TRANSACTIONS OF THE

AMERICAN MATHEMATICAL SOCIETY

Volume 348, Number 6, June 1996

\title{
TOTAL ABSOLUTE CURVATURE AND TIGHTNESS OF NONCOMPACT MANIFOLDS
}

\author{
MARTIN VAN GEMMEREN
}

\begin{abstract}
In the first part we prove an extension of the Chern-Lashof inequality for noncompact immersed manifolds with finitely many ends. For this we give a lower bound of the total absolute curvature in terms of topological invariants of the manifold. In the second part we discuss tightness properties for such immersions. Finally, we give an upper bound for the substantial codimension.
\end{abstract}

\section{INTRODUCTION}

In 1957 S.-s. Chern and R.K. Lashof proved their famous inequality. This inequality states that the total absolute curvature of an immersion of a compact manifold is bounded below by the Morse number which is a differential invariant of the manifold. Further the Morse number is bounded below by the total Betti number of the manifold, a topological invariant [7], [8].

For the first part of this inequality one shows that the total absolute curvature is equal to the expectation value of the number of critical points of a random height function [6], [12]. The second part of the inequality is given by the Morse inequality $[18, \S 5]$.

P. Wintgen [21] showed the Gauß-Bonnet formula for immersions $F: X \rightarrow \mathbb{R}^{m}$, where $X$ has finitely many ends [13] and $F$ is proper with finitely many limit directions and finite total absolute curvature [21]. We shall call such immersions Wintgen immersions.

We want to consider the total absolute curvature of immersions of a noncompact manifold by extending the Chern-Lashof inequality. In the noncompact case the total absolute curvature is still bounded below by the Morse number. But the only topological lower bound of the Morse number tends to be 0 . Then we do not have any information about the relation of critical points of a not necessarily proper nondegenerate function defined on the manifold and the topology of the considered manifold. So we cannot expect a topological lower bound of the Morse number. Therefore we must guarantee that almost every height function is proper in order to establish an extended Chern-Lashof inequality. For this reason we consider Wintgen immersions, since they satisfy this condition. Now we get the (proper) Morse number as a lower bound of the total absolute curvature of a Wintgen immersion.

Received by the editors March 8, 1995.

1991 Mathematics Subject Classification. Primary 53C42; Secondary 57R45.

Key words and phrases. Chern-Lashof inequality, Morse number, ends of manifolds, strong and weak tightness, proper immersion, limit direction.

The author acknowledges support by a fellowship of the Studienstiftung des deutschen Volkes.

(C)1996 American Mathematical Society 
But the (proper) Morse number is not bounded below by the total Betti number. As an example, if the manifold is given by a sphere with three points removed the Morse number is equal to 1 and the total Betti number is equal to 3. For this reason we shall generalize the Morse inequality [18, §5] for (proper) Morse functions of manifolds with finitely many ends. This generalized Morse inequality relates again the Morse number to a topological invariant of the considered manifold.

Equality in the Chern-Lashof inequality for an immersion of a compact manifold holds if and only if for almost every closed halfspace the homomorphism induced by the inclusion into the manifold in homology theory is injective. This is equivalent to the fact that almost every height function has only linking type singularities $[6$, $\S 1.2]$, [19, III 28]. If an immersion satisfies one of these conditions the immersion is called tight. We examine these properties for Wintgen immersions where they now are inequivalent, in general. This yields a definition of tightness for the considered immersions which shall be defined by equality in the generalized Chern-Lashof inequality.

The paper finishes with a theorem about the codimension of tight, substantial Wintgen immersions. In the case of compact manifolds the codimension of 0-tight substantial immersions is bounded by a number which only depends on the dimension of the manifold. Cylinders over substantial not necessarily tight immersions of compact manifolds are simple examples that such a bound cannot exist in the case of noncompact manifolds. The crucial point for this is that in this case the ends are not tightly immersed. If in addition the ends are tightly immersed we can state an upper bound for 0-tight substantial immersions of manifolds with finitely many ends. This bound depends on the dimension of the manifold and the number of ends. Further in certain cases, e.g. for surfaces, we can improve it. But for noncompact manifolds with less than three ends this bound is in any case even smaller than for compact manifolds.

The related topic of taut immersions of noncompact manifolds is considered in [5].

It is my pleasure to thank W. Kühnel for helpful discussions and remarks.

We first recall the topological definition of manifolds with finitely many ends [13]:

Definition 1.1. Let $X$ denote a manifold (or more generally a locally compact Hausdorff space). Let $\mathcal{B} \subseteq \mathcal{P}(X)$ be a base of the topology of $X$ such that for $U, V \in \mathcal{B}:$

- $\partial U$ is compact.

- $U \cup V \in \mathcal{B}$.

- $U \cap V \in \mathcal{B}$.

- $X \backslash \bar{U} \in \mathcal{B}$.

We call a subset $B \subseteq \mathcal{B}$ a $\mathcal{B}$-binding family if every finite sequence $U_{1}, \ldots, U_{n} \in B$ satisfies $\bigcap_{\nu=1}^{n} \bar{U}_{\nu} \neq \emptyset$. We define:

$$
X_{\mathcal{B}}:=\{B \subseteq \mathcal{B} \mid B \text { maximal binding family }\} .
$$

Now

$$
\mathcal{O}_{\mathcal{B}}:=\left\{U_{\mathcal{B}} \subseteq X_{\mathcal{B}} \mid U \in \mathcal{B}\right\}
$$

where

$$
U_{\mathcal{B}}:=\left\{B \in X_{\mathcal{B}} \mid \text { there exists a } V \in B \text { such that } \bar{V} \subseteq U\right\}
$$


is a base for a compact Hausdorff topology on $X_{\mathcal{B}}$ [11], [13].

The mapping

$$
X \rightarrow X_{\mathcal{B}}, \quad x \mapsto\{U \in \mathcal{B} \mid x \in \bar{U}\}
$$

is a topological embedding.

For $\mathcal{F}:=\{U \subseteq X \mid U$ is open, $\partial U$ is compact $\}$ we call $X_{\mathcal{F}}$ the Freudenthal compactification of $X$ and the elements of $X_{\mathcal{F}} \backslash X$ are the ends of $\mathrm{X}$ [13].

For a definition of the Freudenthal compactification by using filters see [14]. For $\mathcal{A}:=\{U \subseteq X \mid U$ is open, $X \backslash U$ is compact $\} X_{\mathcal{A}}$ is the Alexandroff compactification.

Example 1.2. Let $T \subset \mathbb{R}^{3}$ be the solid torus. Then $\mathbb{R}^{3} \backslash T$ is a manifold with two ends. But $\left(\mathbb{R}^{3} \backslash T\right)_{\mathcal{F}}$ is not a manifold.

In the sequel $X$ denotes an $n$-dimensional, smooth manifold with $k$ ends $\infty_{1}, \ldots$, $\infty_{k}, k \in \mathbb{N}$. We assume every considered function $X \rightarrow \mathbb{R}^{m}, m \in \mathbb{N}$ to be smooth. We call such a function proper if the preimages of compact sets are always compact.

Definition 1.3. Let $F: X \rightarrow \mathbb{R}^{m}$ be a proper immersion.

i) The determinant of the shape operator $L: B X \rightarrow \mathbb{R}$ is called the LipschitzKilling curvature defined as a function on the unit normal bundle $B X . L$ is the Gaussian curvature in the case of hypersurfaces.

In the case of orientable $X$ the normalized Lebesgue integral

$$
\operatorname{tac}(X, F):=\frac{1}{c_{m-1}} \int_{B X}|L| d A, \quad c_{m-1}:=\operatorname{Vol}\left(S^{m-1}\right),
$$

where $d A$ is the induced volume element of $B X$, is called the total absolute curvature of $F . \quad S^{m-1}$ denotes the sphere of unit vectors at the origin in $\mathbb{R}^{m}$. For nonorientable $X$ one defines the total absolute curvature by using the orientable double covering. For more details of these definitions see [6, §1.1], [11, §2].

ii) For $e \in S^{m-1}$ we call the mapping

$$
h_{e}: X \rightarrow \mathbb{R}, x \mapsto\langle F(x), e\rangle
$$

the $e$-height function $h_{e}$ with respect to $e$ and $F$.

iii) For $f: X \rightarrow \mathbb{R}, \nu \in \mathbb{N}$ the $\nu^{\text {th }}$ Morse number of $f, \mu_{\nu}(f)$, is defined by $\mu_{\nu}(f):=\{x \in X \mid x$ is a non-degenerate critical point for $f$ of index $\nu\}$.

\section{The Generalized Chern-Lashof inequality}

We define the ends of $X$ with respect to a Morse function on $X$ by the connected components of the subsets below and above the smallest and greatest critical value, respectively. We shall show that the homology of these subsets does not depend on the specific Morse function and that they correspond uniquely to the ends of the considered manifold. This yields a generalized Morse inequality. Hence we can extend the Chern-Lashof inequality.

Proposition 2.1. Let $f: X \rightarrow \mathbb{R}$ be a proper, non-degenerate function with finitely many critical points (a Morse function for short). If $f$ has critical points we denote the smallest critical value by $r_{f} \in \mathbb{R}$, otherwise $r_{f}:=0 . E_{f}:=f^{-1}\left(\left(-\infty, r_{f}\right)\right)$ is called the lower end of $f$. 
i) Every connected component of $X \backslash f^{-1}\left(\left[r_{f}, r_{-f}\right]\right)$ is a punctured neighbourhood of exactly one end. For every end of $X$ there exists exactly one connected component of $X \backslash f^{-1}\left(\left[r_{f}, r_{-f}\right]\right)$ which is a punctured open neighbourhood of this end. We denote this punctured neighbourhood of the end $\infty_{\kappa}$ by $E\left(f, \infty_{\kappa}\right)$.

ii) Let $\left(U_{\nu}\right)$ be a countable base of punctured neighbourhoods of $\infty_{\kappa}$. Let $H_{*}$ be a homology theory. Then

$$
H_{*}\left(E\left(f, \infty_{\kappa}\right)\right) \cong \operatorname{invlim}_{\nu \in \mathbb{N}} H_{*}\left(U_{\nu}\right)=: H_{*}\left(\infty_{\kappa}\right) .
$$

Proof. i) $X \backslash f^{-1}\left(\left[r_{f}, r_{-f}\right]\right)$ is a punctured neighbourhood of every end of $X$ since $f^{-1}\left(\left[r_{f}, r_{-f}\right]\right)$ is compact. $X_{\mathcal{F}}$ is a Hausdorff space. So we can choose disjoint, punctured neighbourhoods $U_{1}, \ldots, U_{k}$ of $\infty_{1}, \ldots, \infty_{k}$, respectively. $X \backslash \bigcup_{\kappa=1}^{k} U_{\kappa}$ is compact.

Since $f$ is proper there exist $r, r^{\prime} \in \mathbb{R}, r<r_{f}, r^{\prime}>r_{-f}$ such that

$$
X \backslash f^{-1}\left(\left(r, r^{\prime}\right)\right) \subseteq X \backslash \bigcup_{\kappa=1}^{k} U_{\kappa}
$$

Hence $X \backslash f^{-1}\left(\left(r, r^{\prime}\right)\right)$ is a strong deformation retract of $X \backslash f^{-1}\left(\left(s, s^{\prime}\right)\right)$ for all $s, s^{\prime} \in \mathbb{R}, r \leq s<r_{f}, r^{\prime} \geq s^{\prime}>r_{-f}[18]$. For this reason $X \backslash f^{-1}\left(\left(r, r^{\prime}\right)\right)$ and $X \backslash f^{-1}\left(\left[r_{f}, r_{-f}\right]\right)$ are of the same singular homology.

ii) For a definition of the inverse limit see [10]. Let $\kappa \in\{1, \ldots, k\}$. We may assume $E\left(f, \infty_{\kappa}\right) \subseteq f^{-1}\left(\left(-\infty, r_{f}\right)\right)$ without loss of generality. Since $\left(U_{\nu}\right)$ is a base of punctured neighbourhoods and $f$ is proper, we can find a subsequence $\left(U_{\nu_{\mu}}\right)$ of $\left(U_{\nu}\right)$ and a sequence $\left(r_{\mu}\right) \in \mathbb{R}^{\mathbb{N}}$ such that

$$
\left(r_{\mu}\right) \rightarrow-\infty \text { and } U_{\nu_{\mu+1}} \subseteq E\left(f, \infty_{\kappa}\right) \cap f^{-1}\left(\left(-\infty, r_{\mu}\right]\right) \subseteq U_{\nu_{\mu}}
$$

Now

$$
H_{*}\left(E\left(f, \infty_{\kappa}\right) \cap f^{-1}\left(\left(-\infty, r_{\mu+1}\right]\right)\right) \cong H_{*}\left(E\left(f, \infty_{\kappa}\right) \cap f^{-1}\left(\left(-\infty, r_{\mu}\right]\right)\right)
$$

induced by the inclusion is an isomorphism. Then the sublevelset $E\left(f, \infty_{\kappa}\right) \cap$ $f^{-1}\left(\left(-\infty, r_{\mu+1}\right]\right)$ is a strong deformation retract of $E\left(f, \infty_{\kappa}\right) \cap f^{-1}\left(\left(-\infty, r_{\mu}\right]\right)[18$, $\S 3]$.

Thus, the mapping

$$
\begin{aligned}
\operatorname{invlim}_{\mu \in \mathbb{N}} H_{*}\left(E\left(f, \infty_{\kappa}\right) \cap f^{-1}\left(\left(-\infty, r_{\mu}\right]\right)\right) & \rightarrow \operatorname{invlim}_{\mu \in \mathbb{N}} H_{*}\left(U_{\nu_{\mu}}\right) \\
\left(x_{\mu}\right) & \mapsto\left(\left(i_{\mu}\right)_{*}\left(x_{\mu}\right)\right)
\end{aligned}
$$

is an isomorphism, where $i_{\mu}: E\left(f, \infty_{\kappa}\right) \cap f^{-1}\left(\left(-\infty, r_{\mu}\right]\right) \rightarrow U_{\nu_{\mu}}$ denotes the inclusion.

By equation $(*)$ we get

$$
H_{*}\left(E\left(f, \infty_{\kappa}\right)\right) \cong \operatorname{invlim}_{\mu \in \mathbf{N}} H_{*}\left(E\left(f ; \infty_{\kappa}\right) \cap f^{-1}\left(\left(-\infty, r_{\mu}\right]\right)\right)
$$

in singular homology. Since the considered subsets are CW-complexes this equation is valid for every homology theory. This proves the assertion.

In the sequel $H_{*}$ denotes a homology theory with a field as coefficent group. We denote by $\beta_{\nu}(X):=\operatorname{dim} H_{\nu}(X), \beta_{\nu}\left(\infty_{\kappa}\right):=\operatorname{dim} H_{\nu}\left(\infty_{\kappa}\right)$ or $\beta_{\nu}(\infty):=\sum_{\kappa=1}^{k} \beta_{\nu}\left(\infty_{\kappa}\right)$ the Betti numbers of $X, \infty_{\kappa}$ or $\infty$, respectively.

We now prove an inequality which enables us to generalize the Chern-Lashof inequality. We shall call this inequality the Morse inequality since it relates the number of critical points of a Morse function to a topological invariant of the manifold. 
Theorem 2.2 (Morse inequality). Let $f: X \rightarrow \mathbb{R}$ be a Morse function. Then

$$
\sum_{\nu=0}^{n} \mu_{\nu}(f) \geq \sum_{\nu=0}^{n}\left|\beta_{\nu}(X)-\frac{1}{2} \beta_{\nu}(\infty)\right|
$$

Proof. Set $\beta_{\nu}\left(X, E_{f}\right):=\operatorname{dim} H_{\nu}\left(X, E_{f}\right)$ for $\nu \in \mathbb{N}$, where $E_{f}$ denotes the lower end of $f$. We now get

$$
\sum_{\nu=0}^{n} \mu_{\nu}(f) \geq \sum_{\nu=0}^{n} \beta_{\nu}\left(X, E_{f}\right)
$$

by the Morse inequality $[18, \S 5]$. The exact homology sequence

$$
\rightarrow H_{\nu}\left(E_{f}\right) \rightarrow H_{\nu}(X) \rightarrow H_{\nu}\left(X, E_{f}\right) \rightarrow H_{\nu-1}\left(E_{f}\right) \rightarrow H_{\nu-1}(X) \rightarrow
$$

gives by consideration of the first or last triple

$$
\begin{gathered}
\beta_{\nu}\left(X, E_{f}\right) \geq \beta_{\nu}(X)-\beta_{\nu}\left(E_{f}\right), \\
\beta_{\nu}\left(X, E_{f}\right) \geq \beta_{\nu-1}\left(E_{f}\right)-\beta_{\nu-1}(X),
\end{gathered}
$$

respectively. By consideration of all five homology groups we get

$$
\beta_{\nu}\left(X, E_{f}\right) \geq \beta_{\nu}(X)-\beta_{\nu}\left(E_{f}\right)-\left(\beta_{\nu-1}(X)-\beta_{\nu-1}\left(E_{f}\right)\right)
$$

for $\nu \in \mathbb{N}$. Now follows

$$
\sum_{\nu=0}^{n} \beta_{\nu}\left(X, E_{f}\right) \geq \sum_{\nu=0}^{n}\left|\beta_{\nu}(X)-\beta_{\nu}\left(E_{f}\right)\right| .
$$

We conclude

$$
\mu(f)=\frac{1}{2}(\mu(f)+\mu(-f)) \geq \sum_{\nu=0}^{n}\left|\beta_{\nu}(X)-\frac{1}{2} \beta_{\nu}(\infty)\right|
$$

by the triangle inequality and Proposition 2.1 .

The crucial point in the proof above is to describe the topology of the ends in terms of the critical points of a Morse function (Proposition 2.1).

Definition 2.3. Let $f: X \rightarrow \mathbb{R}$ be a Morse function. We call $\sum_{\nu=0}^{n} \mu_{\nu}(f)$ the (total) Morse number of $f$. The minimal (total) Morse number of all Morse functions on $X$ is called the Morse number of $X$.

Remark 2.4. The Morse inequality above is only valid for the (total) Morse number of the considered Morse function $f$. Examples for $\mu_{\nu}(f) \nsupseteq\left|\beta_{\nu}(X)-\frac{1}{2} \beta_{\nu}(\infty)\right|$ can easily be given. E.g. we have $\left|\beta_{0}(X)-\frac{1}{2} \beta_{0}(\infty)\right|=\frac{1}{2}$ and $\mu_{0}(X)=0$ if $X$ denotes a sphere with three points removed.

Topologically, the ends of a manifold are something like "topological directions" how the manifold moves to infinity. For submanifolds of a euclidean space one can characterize these directions more analytically by the so-called limit directions [21]:

Definition 2.5. A vector $v \in S^{m-1}$ is called a limit direction with respect to $F$ and $\infty_{\kappa}$ if there exists a sequence $\left(x_{\nu}\right) \in X^{\mathbb{N}}$ such that $\left(x_{\nu}\right) \rightarrow \infty_{\kappa}$ in $X_{\mathcal{F}}$ and $\left(\frac{F\left(x_{\nu}\right)}{\left\|F\left(x_{\nu}\right)\right\|}\right) \rightarrow v$ in $S^{m-1}$. This definition is due to P. Wintgen [21]. 
The concept of limit direction enables us to describe in a more analytical way the property that with respect to an immersion almost every height function is proper. This was done in [21]. Now we can extend the Chern-Lashof inequality in an appropiate way:

Theorem 2.6 (Generalized Chern-Lashof inequality). Let $F: X \rightarrow \mathbb{R}^{m}$ be a proper immersion with finitely many limit directions. Then

$$
\operatorname{tac}(X, F) \geq \mu(X) \geq \sum_{\nu=0}^{n}\left|\beta_{\nu}(X)-\frac{1}{2} \beta_{\nu}(\infty)\right| .
$$

Proof. We may assume without loss of generality the total absolute curvature of $F$ is finite.

We can write

$$
\operatorname{tac}(X, F)=\frac{1}{c_{m-1}} \int_{S^{m-1}} \sum_{\nu=0}^{n} \mu_{\nu}\left(h_{e}\right) d a(e)
$$

as shown in [21]. In particular almost every height function with respect to $F$ is nondegenerate with only finitely many critical points. Since $F$ has only finitely many limit directions almost every height function is proper [21]. Hence almost every height function is a Morse function. By Theorem 2.2 this shows the assertion.

Note that it is meaningful to call the inequality above (extended) Chern-Lashof inequality since $\mu(X)$ is a differential invariant and $\sum_{\nu=0}^{n}\left|\beta_{\nu}(X)-\frac{1}{2} \beta_{\nu}(\infty)\right|$ is a topological invariant. Furthermore for compact manifolds this inequality coincides with the original Chern-Lashof inequality.

\section{Tightness}

P. Wintgen [21] considered immersions $F: X \rightarrow \mathbb{R}^{m}$ of manifolds with finitely many ends, where $F$ is proper with only finitely many limit directions and finite total absolute curvature. He proved the Gauß-Bonnet formula for such immersions. We showed in Section 1 an extension of the Chern-Lashof inequality for such immersions. This is the starting point for a discussion of tightness.

Definition 3.1. We call a proper immersion $F: X \rightarrow \mathbb{R}^{m}$ with only finitely many limit directions and finite total absolute curvature a Wintgen immersion [21]. This definition is given in dedication to P. Wintgen.

One defines tightness for immersions of compact manifolds by using a homology theory $[6, \S 1.5]$. Then an immersion is tight if and only if equality holds in the Chern-Lashof inequality, in other words if and only if almost every height function has only linking type singularities [6, §1.2], [19, III 29]. We examine these properties for Wintgen immersions. This yields three different definitions for tightness of Wintgen immersions, each by using the same (arbitrary) homology theory. One definition is given by generalizing the homology property, one by generalizing the property for critical points of height functions and the most appropriate is given with respect to the generalized Chern-Lashof inequality.

Definition 3.2. Let $F: X \rightarrow \mathbb{R}^{m}$ be a Wintgen immersion. We call $X_{e}^{r}:=$ $h_{e}^{-1}((-\infty, r])$ the sublevelset with respect to $r \in \mathbb{R}$ and $e \in S^{m-1}$. Let $\nu \in \mathbb{N}$. 
i) $F$ is called weak $\nu$-tight if for $0 \leq i \leq \nu$ for almost every $e \in S^{m-1}$

$$
H_{i}\left(X_{e}^{r}, E_{h_{e}}\right) \rightarrow H_{i}\left(X, E_{h_{e}}\right)
$$

is injective for $r \in \mathbb{R}$.

ii) $F$ is called $\nu$-tight if for $0 \leq i \leq \nu$ for almost every $e \in S^{m-1}$

$$
H_{i}\left(X_{e}^{r}\right) \oplus H_{i}\left(X_{-e}^{r}\right) \rightarrow H_{i}\left(X_{e}^{r^{\prime}}\right) \oplus H_{i}\left(X_{-e}^{r^{\prime}}\right)
$$

is injective for every $r, r^{\prime} \in \mathbb{R}, r<r^{\prime}$ or is surjective for every $r, r^{\prime} \in \mathbb{R}, r<r^{\prime}$.

iii) $F$ is called strong $\nu$-tight if for $0 \leq i \leq \nu$ for almost every $e \in S^{m-1}$

$$
H_{i}\left(X_{e}^{r}\right) \rightarrow H_{i}(X)
$$

is injective for $r \in \mathbb{R}$.

iv) We say $F$ immerses $\infty_{\kappa} \nu$-tightly if there exists a $v \in S^{m-1}$ such that $\left\langle v, v_{\kappa}\right\rangle<$ $0, h_{v}$ is a Morse function and $\left.F\right|_{E\left(v, \infty_{\kappa}\right) \cap h_{v_{\kappa}}^{-1}(r)}$ is $\nu$-tight immersion for almost all sufficiently large $r \in \mathbb{R}$.

If $F$ is (weak or strong) $n$-tight we call $F$ (weak or strong) tight for short.

Our next aim is to analyse the relations between these definitions. We shall see that strong tightness implies tightness and tightness implies weak tightness. The converses are not true, in general. But the difference between tightness and strong tightness depends only on the topology of the considered manifold. The difference between tightness and weak tightness is more subtle. One can describe this difference by the measure of bounded height functions if the ends are of the homology of a sphere. In this case one can show that a Wintgen immersion of a manifold with at least two ends is tight if and only if the total absolute curvature is equal to $\beta(X)-2$. The idea can be described as follows: Let $f: X \rightarrow \mathbb{R}$ be a Morse function. If $f$ is bounded (below or above) we get $\beta\left(X, E_{f}\right)=\beta(X)$ and if $f$ is unbounded we get $\beta\left(X, E_{f}\right)=\beta(X)-2$. One needs the Poincaré duality to prove this. So we have to choose a homology theory with coefficents in $\mathbb{Z}_{2}$ to include the non-orientable case. Then, in particular, a Wintgen immersion of a surface with at least two ends is tight if and only if the surface is nowhere positively curved with respect to this immersion. This result follows from the Gauß-Bonnet formula which is proved in [21].

Theorem 3.3. Let $F: X \rightarrow \mathbb{R}^{m}$ be a Wintgen immersion.

i) $F$ is weak tight if and only if $\mu\left(h_{e}\right)=\sum_{\nu=0}^{n} \beta_{\nu}\left(X, E_{h_{e}}\right)$ for almost all $e \in$ $S^{m-1}$.

ii) $F$ is tight if and only if $\mu\left(h_{e}\right)=\sum_{\nu=0}^{n}\left|\beta_{\nu}(X)-\frac{1}{2} \beta_{\nu}(\infty)\right|$ for almost all $e \in S^{m-1}$, so if and only if equality holds in the Chern-Lashof inequality.

iii) $F$ is strong tight if and only if $\mu\left(h_{e}\right)=\sum_{\nu=0}^{n}\left(\beta_{\nu}(X)-\frac{1}{2} \beta_{\nu}(\infty)\right)$ for almost all $e \in S^{m-1}$.

iv) $F$ is strong tight if and only if $F$ is tight and $\beta_{\nu}(X) \geq \frac{1}{2} \beta_{\nu}(\infty)$ for $\nu \in \mathbb{N}$.

v) $F$ is tight if and only if $F$ is weak tight and

$$
\beta\left(X, E_{h_{e}}\right)=\sum_{\nu=0}^{n}\left|\beta_{\nu}(X)-\frac{1}{2} \beta_{\nu}(\infty)\right|
$$

for almost all $e \in S^{m-1}$.

Proof. We only have to modify slightly the proof for the compact case (given in $[6$, $\S 1.3]$, e.g.). The proof is again a computation of critical points of height functions. 
i) The proof is the same as for compact manifolds. We only have to use the theory of linking and non-linking type singularities with respect to relative homology theory [19, III 29] instead of the corresponding theory with respect to simple homology theory in the compact case.

ii) Let $e \in S^{m-1}$ such that $h_{e}$ is a Morse function. We denote the numbers of linking type, non-linking type singularities of index $\nu$ with respect to simple homology theory of $h_{e}$ by $\lambda_{\nu}, \Lambda_{\nu}$, respectively. For a definition of linking and nonlinking type singularity see: [19, III 28], where it is also proved:

$$
\mu_{\nu}\left(h_{e}\right)=\lambda_{\nu}\left(h_{e}\right)+\Lambda_{\nu}\left(h_{e}\right)
$$

and

$$
\beta_{\nu}(X)-\beta_{\nu}\left(E_{h_{e}}\right)=\lambda_{\nu}\left(h_{e}\right)-\Lambda_{\nu+1}\left(h_{e}\right) .
$$

For this reason

$$
\lambda_{\nu}\left(h_{e}\right) \geq \max \left\{0, \beta_{\nu}(X)-\beta_{\nu}\left(E_{h_{e}}\right)\right\}
$$

and

$$
\Lambda_{\nu+1}\left(h_{e}\right) \geq \max \left\{0, \beta_{\nu}\left(E_{h_{e}}\right)-\beta_{\nu}(X)\right\} .
$$

Now we conclude

$$
\mu\left(h_{e}\right) \geq \sum_{\nu=0}^{n}\left|\beta_{\nu}(X)-\beta_{\nu}\left(E_{h_{e}}\right)\right|
$$

where equality holds if and only if

$$
\lambda_{\nu}\left(h_{e}\right)=\max \left\{0, \beta_{\nu}(X)-\beta_{\nu}\left(E_{h_{e}}\right)\right\}
$$

and

$$
\Lambda_{\nu+1}\left(h_{e}\right)=\max \left\{0, \beta_{\nu}\left(E_{h_{e}}\right)-\beta_{\nu}(X)\right\}
$$

for $\nu \in \mathbb{N}$. In other words if and only if

$$
\lambda_{\nu}\left(h_{e}\right)>0 \Rightarrow \Lambda_{\nu+1}\left(h_{e}\right)=0 \text { for } \nu \in \mathbb{N} .
$$

Note this yields another proof of the Morse inequality (Theorem 2.2).

Now let $F: X \rightarrow \mathbb{R}^{m}$ be a Wintgen immersion. We may assume for the sake of simplicity that there is for every critical value of $h_{e}$ only one critical point of $h_{e}$, for almost all $h_{e}$. For this see [16] or more detailed [4].

By the discussion above we get $F$ is tight if and only if

$$
\mu\left(h_{e}\right)=\sum_{\nu=0}^{n}\left|\beta_{\nu}(X)-\beta_{\nu}\left(E_{h_{e}}\right)\right|
$$

and

$$
\left(\beta_{\nu}(X)-\beta_{\nu}\left(E_{h_{e}}\right)\right)\left(\beta_{\nu}(X)-\beta_{\nu}\left(E_{h_{-e}}\right)\right) \geq 0
$$

for almost every $e \in S^{m-1}$. The proof is finished by Proposition 2.1 and the triangle inequality.

iii) The same proof as for the compact case by using Proposition 2.1.

iv) This is a direct consequence of part ii) and iii).

v) This is a direct consequence of part i) and ii). 
In the compact case one says that a 0-tight immersion satisfies the two-piece property. Because in this case an immersion is 0-tight if and only if the preimages with respect to $F$ of almost every halfspace are connected [1] and [6, §1.5]. Evidently we cannot expect the two-piece property (strong 0-tightness) for Wintgen immersions of connected manifolds with more than two ends. Because in this case $\beta_{0}(X)=1<\frac{1}{2} \beta_{0}(\infty)$. The generalization of the two-piece property is in this case 0 -tight, weak 0-tight, respectively.

Proposition 3.4. Let $F: X \rightarrow \mathbb{R}^{m}$ be a Wintgen immersion. Then the following are equivalent:

i) $F$ is weak 0-tight.

ii) Almost every $e \in S^{m-1}$ satisfies: $h_{e}$ has at most one minimum and at most one maximum; $h_{e}$ has a minimum (maximum) if and only if $h_{e}$ is bounded below (above).

Proof. We can carry over the proof for the compact case $[6, \S 1.5]$.

Proposition 3.5. Let $F: X \rightarrow \mathbb{R}^{m}$ be a Wintgen immersion. Then the following are equivalent:

i) $F$ is 0-tight.

ii) For almost all $e \in S^{m-1} h_{e}$ has:

- exactly two extrema if $X$ has no ends,

- exactly one extremum if $X$ has exactly one end,

- no extremum if $X$ has at least two ends.

Proof. This follows by the same methods as for compact manifolds $[6, \S 1.5]$.

\section{The SUbSTANTIAL CODIMENSION}

For the discussion in Section 3 we did not need a correspondence between the (topological) ends of a manifold and their analytical description by limit directions. We only needed to guarantee that almost every height function is proper, a condition which is satisfied by immersions with only finitely many limit directions. But now we want to examine the codimension of substantial 0-tight Wintgen immersions. This requires that there is for every end exactly one limit direction. Wintgen immersions also satisfy this condition as shown below. The proof of this follows immediately from the connectedness of the set of limit directions with respect to one end.

Proposition 4.1. Let $F: X \rightarrow \mathbb{R}^{m}$ be a proper immersion. Then with respect to each end of $X$ the set of limit directions is connected.

Proof. Let $\kappa \in\{1, \ldots, k\}$. Denote the set of limit directions with respect to $\infty_{\kappa}$ by $D_{\kappa}$. For $\epsilon \in \mathbb{R}_{+}$define the open half cone by:

$$
C_{\kappa}(\epsilon):=\left\{r v \mid r \in(1, \infty), v \in S^{m-1}, \operatorname{dist}\left(v, D_{\kappa}\right)<\epsilon\right\} .
$$

By the definition of limit directions the preimmage of every open half cone about $D_{\kappa}$ is a punctured neighbourhood of $\infty_{\kappa}$. If now the compact set $D_{\kappa}$ is disconnected, there exists an open half cone about $D_{\kappa}$ which preimage has at least two unbounded connected components. These components yield to different ends of $X$ by 1.1.; a contradiction. 
Corollary 4.2. Let $F: X \rightarrow \mathbb{R}^{m}$ be a Wintgen immersion. Then there exists for every end $\infty_{\kappa}$ exactly one limit direction with respect to $F$ and $\infty_{\kappa}$. We denote this direction by $v_{\kappa}$.

Proof. Let $\kappa \in\{1, \ldots, k\}$. By the compactness of $S^{m-1}$ there is at least one limit direction for $\infty_{\kappa}$. By the Proposition 4.1 there is at most one.

The following theorem states in particular that in the case of 0-tight Wintgen immersion the ends in the euclidean space have to contract to a single point by moving to infinity if the manifold has at least two ends. More precisely the convex hull of the levelset, $P_{v_{\kappa}}\left(F\left(P_{v_{\kappa}}^{-1}(t)\right)\right)$, with respect to the limit direction $v_{\kappa}$ is increasing. If the manifold has only one end this end has to expand by moving to infinity.

Theorem 4.3. Let $F: X \rightarrow \mathbb{R}^{m}$ be a weak 0-tight Wintgen immersion. Denote the orthogonal projection onto $\left\langle v_{\kappa}\right\rangle^{\perp}$ by $P_{v_{\kappa}}$.

i) We set for $t \in \mathbb{R} P_{v_{\kappa}}^{t}:=P_{v_{\kappa}}\left(F\left(P_{v_{\kappa}}^{-1}(t)\right)\right)$. Then $\operatorname{conv}\left(P_{v_{\kappa}}^{t} \cup P_{v_{\kappa}}^{-t}\right)$ is monotone increasing in $t \in \mathbb{R}$ if no limit direction is orthogonal to $v_{\kappa}$. Further $\operatorname{conv}\left(P_{v_{\kappa}}^{t}\right)$ is monotone increasing in $t \in \mathbb{R}$ if $v_{\kappa}$ closes only positive angles with every other limit direction.

ii) Let $U$ be a punctured neighbourhood of $\infty_{\kappa}$ such that every other end can be separated from $U$. If there exists for almost every $e \in S^{m-1}$ a limit direction which closes a negative angle with e the following equation holds:

$$
\operatorname{conv}\left(P_{v_{\kappa}}(F(U))\right) \subseteq \operatorname{conv}\left(P_{v_{\kappa}}(\partial U)\right) .
$$

Proof. For the idea see also [21].

i) Let $t, t^{\prime} \in \mathbb{R}_{+}, t<t^{\prime}$ and $\kappa \in\{1, \ldots, k\}$ such that

$$
\operatorname{conv}\left(P_{v_{\kappa}}^{t} \cup P_{v_{\kappa}}^{-t}\right) \not \subset \operatorname{conv}\left(P_{v_{\kappa}}^{t^{\prime}} \cup P_{v_{\kappa}}^{-t^{\prime}}\right) \text {. }
$$

We choose

$$
\alpha_{0} \in \operatorname{conv}\left(P_{v_{\kappa}}^{t} \cup P_{v_{\kappa}}^{-t}\right) \backslash \operatorname{conv}\left(P_{v_{\kappa}}^{t^{\prime}} \cup P_{v_{\kappa}}^{-t^{\prime}}\right) .
$$

There exists the perpendicular $\alpha_{\perp}$ of $\alpha_{0} \operatorname{onto} \operatorname{conv}\left(P_{v_{\kappa}}^{t^{\prime}} \cup P_{v_{\kappa}}^{-t^{\prime}}\right)$.

We now show there exists a neighbourhood of $w:=\frac{\alpha_{\perp}-\alpha_{0}}{\left\|\alpha_{\perp}-\alpha_{0}\right\|}$ in $S^{m-1}$ such that every height function with respect to this neighbourhood has a local minimum. This is in contradiction to Proposition 3.4 since $w \perp v_{\kappa}$.

$h_{w}$ has a local minimum. Otherwise for all $s \in \mathbb{R}_{+}$, there exists an $x_{s} \in$ $\partial\left(X_{v_{\kappa}}^{s} \cap X_{-v_{\kappa}}^{s}\right)$ such that $h_{w}\left(x_{s}\right)=\min \left(\left.h_{w}\right|_{X_{v_{\kappa}}^{s} \cap X_{-v_{\kappa}}^{s}}\right)$. Hence we can find a $x_{t^{\prime}} \in h_{w}^{-1}\left(\left\{t^{\prime},-t^{\prime}\right\}\right)$ such that

$$
h_{w}\left(x_{t^{\prime}}\right) \leq h_{w}(x) \text { for all } x \in h_{w}^{-1}\left(\left\{t^{\prime},-t^{\prime}\right\}\right) .
$$

Thus

$$
\left\langle w, P_{v_{\kappa}}\left(F\left(x_{t^{\prime}}\right)\right)\right\rangle \leq\left\langle w, P_{v_{\kappa}}(F(x))\right\rangle \text { for all } x \in h_{w}^{-1}\left(\left\{t^{\prime},-t^{\prime}\right\}\right)
$$

since $w \perp v_{\kappa}$. Finally we get

$$
\left\langle w, P_{v_{\kappa}}\left(F\left(x_{t^{\prime}}\right)\right)\right\rangle \leq\langle w, \alpha\rangle \text { for all } \alpha \in \operatorname{conv}\left(P_{v_{\kappa}}^{t} \cup P_{v_{\kappa}}^{-t}\right) .
$$

In particular $\left\langle w, \alpha_{0}\right\rangle \geq\left\langle w, P_{v_{\kappa}}\left(F\left(x_{t^{\prime}}\right)\right)\right\rangle$.

But we also have $\left\langle w, P_{v_{\kappa}}\left(F\left(x_{t^{\prime}}\right)\right)\right\rangle \geq\left\langle w, \alpha_{\perp}\right\rangle$ since $\alpha_{\perp}$ is the perpendicular of $\alpha_{0}$ onto $\operatorname{conv}\left(P_{v_{\kappa}}^{t^{\prime}} \cup P_{v_{\kappa}}^{-t^{\prime}}\right)$. 
Now it follows $\left\langle w, \alpha_{0}\right\rangle \geq\left\langle w, \alpha_{\perp}\right\rangle$. This implies $-\langle w, w\rangle \geq 0$ in contradiction to the choice of $w$. Since the minimum of $h_{w}$ is local we can find the desired neighbourhood of $w$ in $S^{m-1}([15])$.

ii) $X$ has at least two ends and almost every height function is unbounded by the assumption about the limit directions. So almost every height function has no extremum, since $F$ is weak 0 -tight (Proposition 3.4). Let $U$ be a neighbourhood as in the theorem. Suppose there exists an

$$
\alpha_{0} \in \operatorname{conv}\left(P_{v_{\kappa}}(F(U))\right) \backslash \operatorname{conv}\left(P_{v_{\kappa}}(\partial U)\right) .
$$

Define $\alpha_{\perp}$ to be the perpendicular of $\alpha_{0}$ onto $\operatorname{conv}\left(P_{v_{\kappa}}(F(\partial U))\right)$. One can show in the same way as part i) $\left.h_{w}\right|_{\bar{U}}, w:=\frac{\alpha_{\perp}-\alpha_{0}}{\left\|\alpha_{\perp}-\alpha_{0}\right\|}$ has no minimum which is an element of $\partial U$. Thus there exists a $x_{0} \in U$ such that $h_{w}\left(x_{0}\right)<h_{w}(x)$ for all $x \in \partial U$. Since $\partial U$ is compact $\left.h_{v_{\kappa}}\right|_{\partial U}$ has a global minimum of value $t_{1}$, say. Set $t_{0}:=h_{v_{\kappa}}\left(t_{0}\right)$. Now there exists $t \in \mathbb{R}_{+}$such that

$$
t\left(t_{1}-t_{0}\right)>h_{w}\left(x_{0}\right)-h_{w}(x) \text { for all } x \in \partial U
$$

this means

$$
\left\langle t v_{\kappa}+w, x_{0}\right\rangle<\left\langle t v_{\kappa}+w, x\right\rangle \text { for all } x \in \partial U .
$$

Since $t>0$ we now can find a height function with local minimum in $U$. This finishes the proof as in part i).

Definition 4.4. An immersion $F: X \rightarrow \mathbb{R}^{m}$ is called substantial if $F(X)$ is not contained in an affine hyperplane.

If $X$ is compact the codimension of a 0 -tight, substantial immersion is bounded above by $\frac{n}{2}(n+3)$, where $n$ is the dimension of $X[17],[6, \S 1.5],[12, \S 5]$. There is no such bound for 0 -tight, substantial Wintgen immersions [21]. But if the ends are also 0-tightly immersed the codimension is bounded by a number, which depends on the dimension $n$ and the number of $k$ ends of $X$. Then if the ends are 0tightly immersed they are contained in an affine hypersurface of dimension at most $\frac{n-1}{2}(n+2)$ by the compact case. This enables us to give an upper bound of the substantial codimension for the immersion by the theorem above.

Theorem 4.5. Let $F: X \rightarrow \mathbb{R}^{m}$ be a 0 -tight, substantial Wintgen immersion with 0 -tight immersed ends. Then

$$
m \leq(k-1) \frac{n}{2}(n+1) \text { if } X \text { has at least three ends }
$$

and

$$
m \leq \frac{n}{2}(n+1) \text { if } X \text { has one or two ends. }
$$

Proof. For $\kappa \in\{1, \ldots, k\}$ choose $e_{\kappa} \in S^{m-1},\left\langle v_{\kappa}, e_{\kappa}\right\rangle<0$ such that $h_{e_{\kappa}}$ is a Morse function and $\left.F\right|_{E\left(e_{\kappa}, \infty_{\kappa}\right) \cap h_{v_{\kappa}}^{-1}(r)}$ is a 0-tight immersion for almost all sufficiently large $r \in \mathbb{R}$. Choose $r_{\kappa} \in \mathbb{R}$ of this type. We may assume without loss of generality that $E^{r_{\kappa}}:=E\left(e_{\kappa}, \infty_{\kappa}\right) \cap h_{v_{\kappa}}^{-1}\left(r_{\kappa}\right)$ is an $(n-1)$-dimensional compact manifold. By the codimension theorem for tight immersions of compact manifolds $E^{r_{\kappa}}$ is contained in an affine hyperspace of dimension at most $\frac{n-1}{2}(n+2)$. We choose such an affine subspace $W^{r_{\kappa}}$ with smallest dimension.

Case 1. $X$ has exactly one end. 
Define $W:=\bigcup_{r_{\kappa}}\left\langle v_{\kappa}, W^{r_{\kappa}}\right\rangle_{\text {linear. }}$. (We denote by $\langle,\rangle_{\text {linear }}$ the linear hull.) Then $F(X) \subseteq W$ by Theorem 4.3.i) and $\operatorname{dim}(W) \leq \frac{n-1}{2}(n+2)+1$ since $\left\langle v_{\kappa}, W^{r_{\kappa}}\right\rangle_{\text {linear }} \subseteq$ $\left\langle v_{\kappa}, W^{t^{\prime}}{ }_{\kappa}\right\rangle_{\text {linear }}$ for $t<t^{\prime}$ also by Theorem 4.3.i).

Case 2. $X$ has at least two ends.

The limit directions cannot be linearly independent, because in this case there would exist a set of height functions of positive measure which are bounded below in contradiction to Proposition 3.5. So we may assume without loss of generality $v_{k} \in\left\langle v_{1}, \ldots, v_{k-1}\right\rangle_{\text {linear. }}$. Define $\hat{h}_{v_{\kappa}}:=\left.h_{v_{\kappa}}\right|_{E\left(e_{\kappa}\right)}, U_{\kappa}:=\hat{h}_{v_{\kappa}}^{-1}\left(r_{\kappa}, \infty\right)$.

Now $F\left(U_{\kappa}\right) \subseteq\left\langle v_{\kappa}, W^{r_{\kappa}}\right\rangle_{\text {linear }}$ by Theorem 4.3.ii). Since $X \backslash \overline{\bigcup_{\kappa=1}^{k-1} U_{\kappa}}$ is a punctured neighbourhood of $\infty_{\kappa}$ from which the other ends are separated we get by Theorem 4.3.ii) and the linear dependence of the limit directions:

$$
F(X) \subseteq\left\langle\bigcup_{\kappa=1}^{k-1}\left\langle v_{\kappa}, W^{r_{\kappa}}\right\rangle_{\text {linear }}\right\rangle_{\text {linear }} .
$$

Hence

$$
\begin{aligned}
m & \leq \operatorname{dim}\left(\left\langle\left\{v_{1}, \ldots, v_{k-1}\right\}\right\rangle_{\text {linear }}\right)+(k-1) \frac{n-1}{2}(n+2) \\
& \leq(k-1)\left(\frac{n-1}{2}(n+2)+1\right)=(k-1) \frac{n}{2}(n+1) .
\end{aligned}
$$

Remark 4.6. i) Assume the ends of $X$ have the homology of a sphere. If now $F: X \rightarrow \mathbb{R}^{m}$ is a 0 -tight, substantial Wintgen immersion with tightly immersed ends we get

$$
m \leq(k-1)(n+1) \text { if } X \text { has at least three ends }
$$

and

$$
m \leq n+1 \text { if } X \text { has one or two ends. }
$$

Then by Reeb's theorem $[18, \S 4]$ the ends $E^{r_{\kappa}}$ are spheres. But tightly immersed spheres are substantial hypersurfaces $[6, \S 1.7]$. Note that we have to claim tightly immersed ends since for only 0-tightly immersed ends we cannot conclude they are substantial hypersurfaces [2].

The ends of surfaces are always of spherical homology. So we get

$$
m \leq 3(k-1) \text { if } X \text { has at least three ends }
$$

and

$$
m \leq 3 \text { if } X \text { has one or two ends }
$$

for 0-tight, substantial Wintgen immersions of surfaces $X$ with tightly immersed ends.

ii) An upper bound for the codimension of a 0-tight substantial Wintgen immersion with tightly immersed ends cannot be independent of the number of ends, in general. For this take a tight immersion $f: S^{1} \rightarrow \mathbb{R}^{2}$ such that $\{0\} \times(-2,2) \subset$ $f\left(S^{1}\right)$.

The mapping

$$
F: S^{1} \times \mathbb{R} \rightarrow \mathbb{R}^{3},(x, t) \mapsto(f(x), t)
$$


is a tight Wintgen immersion with tightly immersed ends. Further

$$
F\left(S^{1} \times \mathbb{R}\right) \subset\{0\} \times U:=\{0\} \times(-2,2) \times \mathbb{R} \backslash\{(0,0,0)\} .
$$

By using the mapping

$$
\begin{aligned}
g:\{0\} \times U & \rightarrow \mathbb{R}^{m} \\
(0, x) & \mapsto \begin{cases}(0, x, 0, \ldots, 0, h(x), 0, \ldots, 0), & \text { if } x \in U_{1} ; \\
(0, x, 0, \ldots, 0,0,0, \ldots, 0), & \text { otherwise }\end{cases}
\end{aligned}
$$

where $h: U \rightarrow \mathbb{R}, x \mapsto \exp \left(\frac{2\|x\|^{2}-1}{\left(\|x\|^{2}-1\right)\|x\|^{2}}\right)$ and $U_{1}:=U_{1}((0,0)) \backslash\{(0,0)\}$, one can define by removing $m-3$ points of $S^{1} \times \mathbb{R}$ a substantial, 0-tight Wintgen immersion $G$ with tight ends

$$
G: S^{1} \times \mathbb{R} \backslash\left\{p_{1}, \ldots, p_{m-3}\right\} \rightarrow \mathbb{R}^{m} .
$$

Then with respect to $g$ almost every height function has no extremum since $\exp \left(\frac{2\|x\|^{2}-1}{\left(\|x\|^{2}-1\right)\|x\|^{2}}\right)$ is monotone decreasing with respect to $\|x\|$.

Altogether almost every height function with respect to $G$ has no extremum. Therefore $G$ is 0 -tight (Proposition 3.5). (Indeed $G$ is tight.) Clearly $G$ has tight ends and is substantial.

Remark 4.7. If the number of ends of $X$ is 0 , i.e. if $X$ is compact, the theory above coincides with the usual theory for immersions of compact manifolds.

\section{REFERENCES}

1. T.F. Banchoff, Tightly embedded 2-dimensional polyhedral manifold, Amer. J. Math. 87 (1965), 462-472. MR 31:2729

2. __ High codimensional 0-tight maps on spheres, Proc. Amer. Math. Soc. 161 (1971), 259-267. MR 43:5541

3. D. Braess, Morse-Theorie für berandete Mannigfaltigkeiten, Math. Annalen 208 (1974), 133148. MR 50:1285

4. P. Breuer, Straffe Immersionen von kompakten differenzierbaren Mannigfaltigkeiten in euklidische Räume und die homologische Kennzeichnung dieser Immersionen (insbesondere Zwei-Stück-Eigenschaft), Diploma Thesis, Köln (1990).

5. T.E. Cecil, Taut immersions of non-compact surfaces into a Euclidean 3-space, J. Differential Geometry 11 (1976), 451-459. MR 55:11275

6. T.E. Cecil and P.J. Ryan, Tight and Taut Immersions of Manifolds, Pitman, London, 1985. MR 87b:53089

7. S.-s. Chern and R.K. Lashof, On the total curvature of immersed manifolds. I, Amer. J. Math. 79 (1957), 396-398. MR 18:927a

8. 20:4301

9. A. Dold, Lectures on Algebraic Topology, Springer, Berlin, 1980. MR 82c:55001

10. S. Eilenberg and N. Steenrod, Foundations of algebraic topology, Princeton University Press, Princeton, 1952. MR 14:398b

11. Ky Fan and N. Gottesman, On compactifications of Freudenthal and Wallman, Indag. Math. 14 (1952), 504-510. MR 14:669c

12. D. Ferus, Totale Absolutkrümmung in Differentialgeometrie und-topologie, Lecture Notes in Mathematics 66, Springer, 1968. MR 40:3468

13. H. Freudenthal, Kompaktifizierungen und Bikompaktifizierungen, Indag. Math. 13 (1951), 184-192. MR 12:728b

14. C.H. Houghton, Ends of locally compact groups and their coset spaces, J. Australian Math. Soc. 17 (1974), 274-284. MR 50:10147 
15. N.H. Kuiper, On surfaces in euclidean three spaces, Bull. Soc. Math. Belg. 12 (1960), 5-22. MR 23:A609

16. — On convex maps, Nieuv Archief voor Wisk. 10 (1962), 147-164. MR 26:3076

17. Minimal total absolute curvature for immersions, Invent. Math. 10 (1970), 209-238. MR 42:2499

18. J. Milnor, Morse Theory, Annals of Mathematical Studies 51, Princeton, 1963. MR 29:634

19. M. Morse and S.S. Cairns, Critical point theory in global analysis and differential topology, Academic Press, New York, 1969. MR 39:6358

20. R. Sulanke und P. Wintgen, Differentialgeometrie und Faserbündel, Birkhäuser, Berlin-BaselStuttgart, 1972. MR 54:1274

21. P. Wintgen, On total absolute curvature of nonclosed submanifolds, Annals of Global Analysis and Geometry 2 (1984), 55-87. MR 85m:53071

Mathematisches Institut B, Universität Stuttgart, 70550 Stuttgart, Germany

E-mail address: mvg@mathematik.uni-stuttgart.de 